Article

\title{
Some Integral Inequalities for $h$-Godunova-Levin Preinvexity
}

\author{
Ohud Almutairi ${ }^{1,+}$ and Adem Kılıçman ${ }^{2, *,+}$ (D) \\ 1 Department of Mathematics, University of Hafr Al-Batin, Hafr Al-Batin 31991, Saudi Arabia, \\ AhoudbAlmutairi@gmail.com \\ 2 Department of Mathematics, Universiti Putra Malaysia, Serdang 43400, Malaysia \\ * Correspondence: akilic@upm.edu.my; Tel.: +603-89466813 \\ + These authors contributed equally to this work.
}

Received: 15 November 2019; Accepted: 9 December 2019; Published: 11 December 2019

\begin{abstract}
In this study, we define new classes of convexity called $h$-Godunova-Levin and $h$-Godunova-Levin preinvexity, through which some new inequalities of Hermite-Hadamard type are established. These new classes are the generalization of several known convexities including the $s$-convex, $P$-function, and Godunova-Levin. Further, the properties of the $h$-Godunova-Levin function are also discussed. Meanwhile, the applications of $h$-Godunova-Levin Preinvex function are given.
\end{abstract}

Keywords: Hermite-Hadamard inequality; $h$-convexity; $h$-Godunova-Levin function; $h$-Godunova-Levin preinvex function

\section{Introduction}

Recently, the theory of convexity has become a broad area of study since it is related to the theory of inequalities. Many such inequalities are frequently reported in the literature as a result of applications of convexity in both pure and applied sciences (see [1-4]). Considering its many applications in different branches of mathematics, convexity can provide a basis for estimating error bounds in a large class of problems [5]. One example of these is how the convexity was applied to estimate errors when using a trapezoidal formula for numerical integration [6,7]. Others include studying problems in nonlinear programming and applying them to special means [8]. Among them, an interesting inequality for convex function is of Hermite-Hadamard type, which can be stated as follows:

Let $S$ be a nonempty subset in $\mathbb{R}, \psi: S \rightarrow \mathbb{R}$ be a convex function on $S$, and $u_{1}, u_{2} \in S, u_{1}<u_{2}$, then we have

$$
\psi\left(\frac{u_{1}+u_{2}}{2}\right) \leq \frac{1}{u_{2}-u_{1}} \int_{u_{1}}^{u_{2}} \psi(x) d x \leq \frac{\psi\left(u_{1}\right)+\psi\left(u_{2}\right)}{2} .
$$

If $\psi$ is a concave function, the two inequalities can be held in the reverse direction. These inequalities have been extensively improved and generalized. For example, see [1,9-12].

Definition 1. [13] A positive function $\psi: S \subseteq \mathbb{R} \rightarrow \mathbb{R}$ is said to be a Godunova-Levin if

$$
\psi\left(\delta u_{1}+(1-\delta) u_{2}\right) \leq \frac{\psi\left(u_{1}\right)}{\delta}+\frac{\psi\left(u_{2}\right)}{1-\delta}, \forall u_{1}, u_{2} \in S, \delta \in(0,1) .
$$

Several other properties related to this class of functions are given in [14-16]. For example, both the positive monotone and positive convex functions belong to this class. 
This concept has been recently extended to $s$-Godunova-Levin type of convexity by Dragomir [17]. Furthermore, studies were conducted on s-Godunova-Levin type convexity and can be found in the literature [6]. Another important class of convex function is $h$-convexity, which was introduced by Varošanec [18], through which several generalizations and extensions were made.

Definition 2. [18] Let $\psi, \omega: S \subseteq \mathbb{R} \rightarrow \mathbb{R}$ be two functions, such that $u_{1}, u_{2} \in S$, the inequality $\left(\psi\left(u_{1}\right)-\right.$ $\left.\psi\left(u_{2}\right)\right)\left(\omega\left(u_{1}\right)-\omega\left(u_{2}\right)\right) \geq 0$ is called similarly ordered for $\psi$ and $\omega$ on $S$.

Now, the following preliminaries on invexity analysis are necessary since they can be frequently used throughout this study. Therefore, we let $S$ be the nonempty subset in $\mathbb{R}$ and let $\psi: S \rightarrow \mathbb{R}$ and $\zeta(.,):. S \times S \rightarrow \mathbb{R}$ be a continuous function.

Definition 3. $[19,20] A$ set $S$ is said to be an invex set with respect to $\zeta(.,$.$) if, for every u_{1}, u_{2} \in S$, and $\delta \in[0,1]$

$$
u_{1}+\delta \zeta\left(u_{2}, u_{1}\right) \in S
$$

Definition 4. [20] A function $\psi$ on the invex set $S$ is said to be preinvex with respect to $\zeta$ if

$$
\psi\left(u_{1}+\delta \zeta\left(u_{2}, u_{1}\right)\right) \leq(1-\delta) \psi\left(u_{1}\right)+\delta \psi\left(u_{2}\right) ; \forall u_{1}, u_{2} \in S ; \delta \in[0,1] .
$$

Usually, the preinvex functions can be convexity if $\zeta\left(u_{2}, u_{1}\right)=u_{2}-u_{1}$ holds in (2). Other properties of preinvex functions are given in [21,22].

We arrange this paper as follows. Section 2 introduces the new classes of $h$-Godunova-Levin, denoted by $\operatorname{SGX}\left(\frac{1}{h}, t\right)$ and $S G V\left(\frac{1}{h}, t\right)$, together with their properties. This class of function unifies different classes of convexity: s-Godunova-Levin, P-functions, s-convexity, and Godunova-Levin. In Section 3, we prove new Hermite-Hadamard inequalities via $h$-Godunova-Levin preinvexity. Section 4 introduces a new definition of $h$-Godunova-Levin preinvexity, which can be the generalization of preinvexity. This Section also presents new Hermite-Hadamard type inequalities for $h$-Godunova-Levin preinvexity. Section 5 gives some applications to special means, as well as an application to numerical integration.

\section{The $h$-Godunova-Levin Functions and Their Properties}

This section introduces the notion of $h$-Godunova-Levin function together with their properties. This class of function can be denoted by $S G X\left(\frac{1}{h}, t\right)$ and $S G V\left(\frac{1}{h}, t\right)$ for $h$-Godunova-Levin convex and $h$-Godunova-Levin concave, respectively.

Definition 5. Suppose $h:(0,1) \rightarrow \mathbb{R}$. A non-negative function $\psi: S \rightarrow \mathbb{R}$ is said to be h-Godunova-Levin, or that $\psi$ belongs to the class $S G X\left(\frac{1}{h}, S\right)$, for all $u_{1}, u_{2} \in S$ and $\delta \in(0,1)$, we have

$$
\psi\left(\delta u_{1}+(1-\delta) u_{2}\right) \leq \frac{\psi\left(u_{1}\right)}{h(\delta)}+\frac{\psi\left(u_{2}\right)}{h(1-\delta)} .
$$

Remark 1. If $h(\delta)=\delta, h(\delta)=\frac{1}{\delta}, h(\delta)=\delta^{s}, h(\delta)=1, h(\delta)=\frac{1}{\delta^{s}}$ in inequality (3), the definition of $h$-Godunova-Levin function can be clearly reduced to different types of convexity, such as Godunova-Levin function, classical convex, s-Godunova-Levin function, P-function, and s-convex function. This indicates that $h$-Godunova-Levin function is the generalization of these different classes. 
Proposition 1. Suppose that $h_{1}, h_{2}$ are two positive functions defined on the interval $S$ satisfying the property

$$
\frac{1}{h_{1}(\delta)} \leq \frac{1}{h_{2}(\delta)}, \delta \in(0,1) .
$$

If $\psi \in S G X\left(\frac{1}{h_{1}}, S\right)$, then $\psi \in S G X\left(\frac{1}{h_{2}}, S\right)$. If $\psi \in S G V\left(\frac{1}{h_{1}}, S\right)$, then $\psi \in S G V\left(\frac{1}{h_{2}}, S\right)$, where $h_{1}(t) \neq 0$ and $h_{2}(t) \neq 0$.

Proof. If $\psi \in S G X\left(\frac{1}{h_{1}}, S\right)$, then for any $u_{1}, u_{2} \in S$ and $\delta \in(0,1)$ we get

$$
\begin{aligned}
\psi\left(\delta u_{1}+(1-\delta) u_{2}\right) & \leq \frac{1}{h_{1}(\delta)} \psi\left(u_{1}\right)+\frac{1}{h_{1}(1-\delta)} \psi\left(u_{2}\right) \\
& \leq \frac{1}{h_{2}(\delta)} \psi\left(u_{1}\right)+\frac{1}{h_{2}(1-\delta)} \psi\left(u_{2}\right)
\end{aligned}
$$

i.e., $\psi \in S G X\left(\frac{1}{h_{2}}, S\right)$.

Proposition 2. If $\psi, \omega \in S G X\left(\frac{1}{h}, S\right)$ and $\lambda>0$, then $\psi+\omega, \lambda \psi \in S G X\left(\frac{1}{h}, S\right)$. If $\psi, \omega \in S G V\left(\frac{1}{h}, S\right)$ and $\lambda>0$, then $\psi+\omega, \lambda \psi \in \operatorname{SGV}\left(\frac{1}{h}, S\right)$.

Proof. The proof is clear from the definition of the classes $h$-Godunova-Levin convex and $h$-Godunova-Levin concave, $S G X\left(\frac{1}{h}, S\right)$ and $S G V\left(\frac{1}{h}, S\right)$, respectively.

Proposition 3. Suppose that $\psi$ and $\omega$ are two h-Godunova-Levin functions and satisfying the property given in Definition 2. Then, the product of these two functions satisfies

$$
\psi\left(\delta u_{1}+(1-\delta) u_{2}\right) \omega\left(\delta u_{1}+(1-\delta) u_{2}\right) \leq\left[\frac{1}{h(\delta)} \psi\left(u_{1}\right) \omega\left(u_{1}\right)+\frac{1}{h(1-\delta)} \psi\left(u_{2}\right) \omega\left(u_{2}\right)\right]\left[\frac{1}{h(\delta)}+\frac{1}{h(1-\delta)}\right] .
$$

Proof. Given that $\psi$ and $\omega$ are $h$-Godunova-Levin functions, we have

$$
\begin{aligned}
\psi\left(\delta u_{1}+(1-\delta) u_{2}\right) \omega\left(\delta u_{1}+(1-\delta) u_{2}\right) \leq & \left(\frac{\psi\left(u_{1}\right)}{h(\delta)}+\frac{\psi\left(u_{2}\right)}{h(1-\delta)}\right)\left(\frac{\omega\left(u_{1}\right)}{h(\delta)}+\frac{\omega\left(u_{2}\right)}{h(1-\delta)}\right) \\
= & \frac{1}{(h(\delta))^{2}} \psi\left(u_{1}\right) \omega\left(u_{1}\right)+\frac{1}{h(\delta) h(1-t)}\left[\psi\left(u_{1}\right) \omega\left(u_{2}\right)\right. \\
& \left.+\psi\left(u_{2}\right) \omega\left(u_{1}\right)\right]+\frac{1}{(h(1-\delta))^{2}} \psi\left(u_{2}\right) \omega\left(u_{2}\right) \\
= & {\left[\frac{1}{h(\delta)} \psi\left(u_{1}\right) \omega\left(u_{1}\right)+\frac{1}{h(1-\delta)} \psi\left(u_{2}\right) \omega\left(u_{2}\right)\right]\left[\frac{1}{h(\delta)}+\frac{1}{h(1-\delta)}\right] . }
\end{aligned}
$$

Proposition 4. Suppose that $\psi: S_{1} \rightarrow[0, \infty), \omega: S_{2} \rightarrow[0, \infty)$ are two functions such that $\omega\left(S_{2}\right) \subseteq S_{1}$. If the function $\omega$ is convex (concave), and the function $\psi$ is increasing (decreasing), $\psi \in S G X\left(\frac{1}{h}, S_{1}\right)$, then the composition $\psi \circ \omega$ belongs to $\operatorname{SGX}\left(\frac{1}{h}, S_{2}\right)$. Meanwhile, if the function $\omega$ is convex (concave) and the function $\psi$ is decreasing (increasing), $\psi \in S G V\left(\frac{1}{h}, S_{1}\right)$, then the composition $\psi \circ \omega$ belongs to $S G V\left(\frac{1}{h}, S_{2}\right)$.

Proof. Suppose that $\omega$ is a convex function, $\psi$ is increasing, and $\psi \in \operatorname{SGX}\left(\frac{1}{h}, S_{1}\right)$. Then, we have

$$
(\psi \circ \omega)\left(\left(\delta u_{1}+(1-\delta) u_{2}\right)\right) \leq \psi\left(\delta \omega\left(u_{1}\right)+(1-\delta) \omega\left(u_{2}\right)\right) \leq \frac{1}{h(\delta)}(\psi \circ \omega)\left(u_{1}\right)+\frac{1}{h(1-\delta)}(\psi \circ \omega)\left(u_{2}\right),
$$

for all $u_{1}, u_{2} \in S_{2}$, and $\delta \in(0,1)$. 


\section{New Hermite-Hadamard Inequality for $h$-Godunova-Levin Convex Function}

The following generalization of the Hermite-Hadamard inequalities for $h$-Godunova-Levin convex function can be proved in this section.

Theorem 1. Let $\psi \in S G X\left(\frac{1}{h}, S\right), u_{1}, u_{2} \in S$, with $u_{1}<u_{2}$ and $\psi \in L_{1}\left(\left[u_{1}, u_{2}\right]\right)$, where $h:(0,1) \rightarrow \mathbb{R}$ is a positive function and $h(\delta) \neq 0$, we have

$$
\frac{h\left(\frac{1}{2}\right)}{2} \psi\left(\frac{u_{1}+u_{2}}{2}\right) \leq \frac{1}{u_{2}-u_{1}} \int_{u_{1}}^{u_{2}} \psi(x) d x \leq\left[\psi\left(u_{1}\right)+\psi\left(u_{2}\right)\right] \int_{0}^{1} \frac{1}{h(\delta)} d \delta .
$$

Proof. Since $\psi$ is $h$-Godunova-Levin, we have

$$
\psi\left(\delta u_{1}+(1-\delta) u_{2}\right) \leq \frac{\psi\left(u_{1}\right)}{h(\delta)}+\frac{\psi\left(u_{2}\right)}{h(1-\delta)}
$$

Considering $v_{1}=\alpha u_{1}+(1-\alpha) u_{2}, v_{2}=(1-\alpha) u_{1}+\alpha u_{2}$, and $\delta=\frac{1}{2}$ in (5), we obtain

$$
\begin{aligned}
\psi\left(\frac{u_{1}+u_{2}}{2}\right) & \leq \frac{1}{h\left(\frac{1}{2}\right)} \psi\left(\alpha u_{1}+(1-\alpha) u_{2}\right)+\frac{1}{h\left(\frac{1}{2}\right)} \psi\left((1-\alpha) u_{1}+\alpha u_{2}\right) \\
& \leq \frac{1}{h\left(\frac{1}{2}\right)}\left[\psi\left(\alpha u_{1}+(1-\alpha) u_{2}\right)+\psi\left((1-\alpha) u_{1}+\alpha u_{2}\right)\right] .
\end{aligned}
$$

Thus, after integrating (6), we get the following

$$
\begin{aligned}
\psi\left(\frac{u_{1}+u_{2}}{2}\right) & \leq \frac{1}{h\left(\frac{1}{2}\right)}\left[\int_{0}^{1} \psi\left(\alpha u_{1}+(1-\alpha) u_{2}\right) d \alpha+\int_{0}^{1} \psi\left((1-\alpha) u_{1}+\alpha u_{2}\right) d \alpha\right] \\
& \leq \frac{2}{h\left(\frac{1}{2}\right)\left(u_{2}-u_{1}\right)} \int_{u_{1}}^{u_{2}} \psi(x) d x
\end{aligned}
$$

This ends the proof of the first inequality. Now, taking $v_{1}=u_{1}$ and $v_{2}=u_{2}$ in (5) and integrating the result over the interval $[0,1]$ with respect to $\delta$, we obtain

$$
\frac{1}{u_{2}-u_{1}} \int_{u_{1}}^{u_{2}} \psi(x) d x \leq\left[\psi\left(u_{1}\right)+\psi\left(u_{2}\right)\right] \int_{0}^{1} \frac{1}{h(\delta)} d \delta .
$$

This completes the proof of the second inequality (4).

Remark 2. In Theorem 1, choosing $h(\delta)=\delta^{s}$, we obtain the Hermite-Hadamard inequalities for s-convexity in the second sense, Theorem 2.1. in [23]. If we choose $h(\delta)=1$, Theorem 1 can be reduced to the result for P-function [12]. Taking $h(\delta)=\frac{1}{\delta}$, the theorem reduces the result for classical Hermite-Hadamard inequalities given in inequality (1).

\section{Hermite-Hadamard Inequalities for $h$-Godunova-Levin Preinvex Function}

The definition of $h$-Godunova-Levin preinvex is introduced in this section. The inequalities of Hermite-Hadamard type for functions whose first derivatives absolute values are $h$-Godunova-Levin preinvex are also presented here.

Definition 6. A function $\psi: S \rightarrow \mathbb{R}$ is said to be $h$-Godunova-Levin preinvex function with respect to $\zeta$ if, for all $u_{1}, u_{2} \in S, \delta \in(0,1)$,

$$
\psi\left(u_{1}+\delta \zeta\left(u_{2}, u_{1}\right)\right) \leq \frac{\psi\left(u_{1}\right)}{h(1-\delta)}+\frac{\psi\left(u_{2}\right)}{h(\delta)}
$$


holds.

The following lemma can be used to prove the generalization of the Hermite-Hadamard inequalities for $h$-Godunova-Levin preinvexity.

Lemma 1. [24] Suppose that $\psi: S=\left[u_{1}, u_{1}+\zeta\left(u_{2}, u_{1}\right)\right] \rightarrow(0, \infty)$ is a differentiable function, where $u_{1}, u_{1}+\zeta\left(u_{2}, u_{1}\right) \in S$ with $u_{1}<u_{1}+\zeta\left(u_{2}, u_{1}\right)$. If $\psi^{\prime} \in L 1\left[u_{1}, u_{1}+\zeta\left(u_{2}, u_{1}\right)\right]$, we have

$$
\frac{1}{\zeta\left(u_{2}, u_{1}\right)} \int_{u_{1}}^{u_{1}+\zeta\left(u_{2}, u_{1}\right)} \psi(x) d x-\frac{\psi\left(u_{1}\right)+\psi\left(u_{1}+\zeta\left(u_{2}, u_{1}\right)\right)}{2}=\frac{\zeta\left(u_{2}, u_{1}\right)}{2}\left[\int_{0}^{1}(1-2 t) \psi^{\prime}\left(u_{1}+\delta \zeta\left(u_{2}, u_{1}\right)\right) d \delta\right] .
$$

Theorem 2. Suppose that $\psi: S=\left[u_{1}, u_{1}+\zeta\left(u_{2}, u_{1}\right)\right] \rightarrow(0, \infty)$ is a differentiable mapping on $S^{\circ}$, $u_{1}, \zeta\left(u_{2}, u_{1}\right) \in S^{\circ}$, with $u_{1}<u_{1}+\zeta\left(u_{2}, u_{1}\right)$. If $\left|\psi^{\prime}\right|$ is a h-Godunova-Levin preinvex on $\left[u_{1}, u_{1}+\zeta\left(u_{2}, u_{1}\right)\right]$, then we get the following inequality:

$$
\begin{aligned}
\left|\frac{\psi\left(u_{1}\right)+\psi\left(u_{1}+\zeta\left(u_{2}, u_{1}\right)\right)}{2}-\frac{1}{\zeta\left(u_{2}, u_{1}\right)} \int_{u_{1}}^{u_{1}+\zeta\left(u_{2}, u_{1}\right)} \psi(x) d x\right| & \leq \frac{\zeta\left(u_{2}, u_{1}\right)}{2}\left[\left|\psi^{\prime}\left(u_{1}\right)\right|+\left|\psi^{\prime}\left(u_{2}\right)\right|\right] \\
& \times \int_{0}^{1}|1-2 \delta|\left[\frac{1}{h(\delta)}+\frac{1}{h(1-\delta)}\right] d \delta .
\end{aligned}
$$

Proof. We use Lemma 1 to prove inequality (7) as follows:

$$
\begin{aligned}
\left|\frac{\psi\left(u_{1}\right)+\psi\left(u_{1}+\zeta\left(u_{2}, u_{1}\right)\right)}{2}-\frac{1}{\zeta\left(u_{2}, u_{1}\right)} \int_{u_{1}}^{u_{1}+\zeta\left(u_{2}, u_{1}\right)} \psi(x) d x\right| & =\left|\frac{\zeta\left(u_{2}, u_{1}\right)}{2} \int_{0}^{1}(1-2 \delta) \psi^{\prime}\left(u_{1}+\delta \zeta\left(u_{2}, u_{1}\right)\right) d \delta\right| \\
& \leq \frac{\zeta\left(u_{2}, u_{1}\right)}{2} \int_{0}^{1}|1-2 \delta|\left|\psi^{\prime}\left(u_{1}+\delta \zeta\left(u_{2}, u_{1}\right)\right) d \delta\right| \\
& \leq \frac{\zeta\left(u_{2}, u_{1}\right)}{2} \int_{0}^{1}|1-2 \delta|\left|\frac{\psi^{\prime}\left(u_{1}\right)}{h(\delta)}+\frac{\psi^{\prime}\left(u_{2}\right)}{h(1-\delta)}\right| d \delta \\
& \leq \frac{\zeta\left(u_{2}, u_{1}\right)}{2}\left[\left|\psi^{\prime}\left(u_{1}\right)\right|+\left|\psi^{\prime}\left(u_{2}\right)\right|\right] \\
& \times \int_{0}^{1}|1-2 \delta|\left[\frac{1}{h(\delta)}+\frac{1}{h(1-\delta)}\right] d \delta .
\end{aligned}
$$

Corollary 1. Since $\int_{0}^{1} \frac{1}{h(1-\delta)} d \delta=\int_{0}^{1} \frac{1}{h(\delta)} d \delta$, subsituting this fact in inequality (7), we get

$$
\begin{aligned}
\left|\frac{\psi\left(u_{1}\right)+\psi\left(u_{1}+\zeta\left(u_{2}, u_{1}\right)\right)}{2}-\frac{1}{\zeta\left(u_{2}, u_{1}\right)} \int_{u_{1}}^{u_{1}+\zeta\left(u_{2}, u_{1}\right)} \psi(x) d x\right| \leq & \frac{\zeta\left(u_{2}, u_{1}\right)}{2}\left[\left|\psi^{\prime}\left(u_{1}\right)\right|+\left|\psi^{\prime}\left(u_{2}\right)\right|\right] \\
& \times \int_{0}^{1} \frac{|1-2 \delta|}{h(\delta)} d \delta .
\end{aligned}
$$

Corollary 2. Taking $\zeta\left(u_{2}, u_{1}\right)=u_{2}-u_{1}$ in inequality (8), we obtain the following inequality:

$$
\left|\frac{\psi\left(u_{1}\right)+\psi\left(u_{2}\right)}{2}-\frac{1}{u_{2}-u_{1}} \int_{u_{1}}^{u_{2}} \psi(x) d x\right| \leq \frac{u_{2}-u_{1}}{2}\left[\left|\psi^{\prime}\left(u_{1}\right)\right|+\left|\psi^{\prime}\left(u_{2}\right)\right|\right] \int_{0}^{1} \frac{|1-2 \delta|}{h(\delta)} d \delta .
$$

Theorem 3. Suppose that $\psi: S=\left[u_{1}, u_{1}+\zeta\left(u_{2}, u_{1}\right)\right] \rightarrow(0, \infty)$ is a differentiable function on $S^{\circ}$, $u_{1}, \zeta\left(u_{2}, u_{1}\right) \in S^{\circ}$, with $u_{1}<u_{1}+\zeta\left(u_{2}, u_{1}\right)$. If $\left|\psi^{\prime}\right|$ is a h-Godunova-Levin preinvex on $\left[u_{1}, u_{1}+\zeta\left(u_{2}, u_{1}\right)\right]$, 
with $p>1$ such that $q=\frac{p}{p-1}$, we obtain

$$
\begin{aligned}
\left|\frac{\psi\left(u_{1}\right)+\psi\left(u_{1}+\zeta\left(u_{2}, u_{1}\right)\right)}{2}-\frac{1}{\zeta\left(u_{2}, u_{1}\right)} \int_{u_{1}}^{u_{1}+\zeta\left(u_{2}, u_{1}\right)} \psi(x) d x\right| \leq & \frac{\zeta\left(u_{2}, u_{1}\right)}{(p+1)^{\frac{1}{p}}}\left(\left|\psi^{\prime}\left(u_{1}\right)\right|^{q}\right. \\
& \left.+\left|\psi^{\prime}\left(u_{2}\right)\right|^{q}\right)^{\frac{1}{q}} \int_{0}^{1} \frac{1}{h(\delta)} d \delta .
\end{aligned}
$$

Proof. Applying Lemma 1, we have

$$
\begin{aligned}
\left|\frac{\psi\left(u_{1}\right)+\psi\left(u_{1}+\zeta\left(u_{2}, u_{1}\right)\right)}{2}-\frac{1}{\zeta\left(u_{2}, u_{1}\right)} \int_{u_{1}}^{u_{1}+\zeta\left(u_{2}, u_{1}\right)} \psi(x) d x\right| & =\left|\frac{\zeta\left(u_{2}, u_{1}\right)}{2} \int_{0}^{1}(1-2 \delta) \psi^{\prime}\left(u_{1}+\delta \zeta\left(u_{2}, u_{1}\right)\right) d \delta\right| \\
& \leq \frac{\zeta\left(u_{2}, u_{1}\right)}{2} \int_{0}^{1}|1-2 \delta|\left|\psi^{\prime}\left(u_{1}+\delta \zeta\left(u_{2}, u_{1}\right)\right) d \delta\right| .
\end{aligned}
$$

We use Hölder's integral inequality as follows:

$$
\begin{aligned}
\left|\frac{\psi\left(u_{1}\right)+\psi\left(u_{1}+\zeta\left(u_{2}, u_{1}\right)\right)}{2}-\frac{1}{\zeta\left(u_{2}, u_{1}\right)} \int_{u_{1}}^{u_{1}+\zeta\left(u_{2}, u_{1}\right)} \psi(x) d x\right| & \leq \frac{\zeta\left(u_{2}, u_{1}\right)}{2}\left(\int_{0}^{1}|1-2 \delta|^{p} d \delta\right)^{\frac{1}{p}} \\
& \times\left(\int_{0}^{1}\left|\psi^{\prime}\left(u_{1}+\delta \zeta\left(u_{2}, u_{1}\right)\right)\right|^{q} d \delta\right)^{\frac{1}{q}}
\end{aligned}
$$

where $\frac{1}{p}+\frac{1}{q}=1$.

Now, since $\left|\psi^{\prime}\right|^{p}$ is a $h$-Godunova-Levin preinvex, we obtain

$$
\begin{aligned}
\int_{0}^{1}\left|\psi^{\prime}\left(u_{1}+\delta \zeta\left(u_{2}, u_{1}\right)\right)\right|^{q} d \delta & \leq \int_{0}^{1}\left(\frac{\left|\psi^{\prime}\left(u_{1}\right)\right|^{q}}{h(\delta)}+\frac{\left|\psi^{\prime}\left(u_{2}\right)\right|^{q}}{h(1-\delta)}\right) d \delta \\
& \leq 2 \int_{0}^{1} \frac{1}{h(\delta)} d \delta\left(\left|\psi^{\prime}\left(u_{1}\right)\right|^{q}+\left|\psi^{\prime}\left(u_{2}\right)\right|^{q}\right) .
\end{aligned}
$$

Using the basic calculus, we have $\int_{0}^{1}|1-2 \delta|^{p} d \delta=\frac{1}{p+1}$. This completes the proof of the Theorem 3.

Corollary 3. Choosing $\zeta\left(u_{2}, u_{1}\right)=u_{2}-u_{1}$ in Theorem 3 reduces inequality (9) to the following:

$$
\left|\frac{\psi\left(u_{1}\right)+\psi\left(u_{2}\right)}{2}-\frac{1}{u_{2}-u_{1}} \int_{u_{1}}^{u_{2}} \psi(x) d x\right| \leq \frac{u_{2}-u_{1}}{(p+1)^{\frac{1}{p}}}\left(\left|\psi^{\prime}\left(u_{1}\right)\right|^{q}+\left|\psi^{\prime}\left(u_{2}\right)\right|^{q}\right)^{\frac{1}{q}} \int_{0}^{1} \frac{1}{h(\delta)} d \delta .
$$

Theorem 4. With the assumptions of Theorem 3, we get the following:

$$
\begin{aligned}
\left|\frac{\psi\left(u_{1}\right)+\psi\left(u_{1}+\zeta\left(u_{2}, u_{1}\right)\right)}{2}-\frac{1}{\zeta\left(u_{2}, u_{1}\right)} \int_{u_{1}}^{u_{1}+\zeta\left(u_{2}, u_{1}\right)} \psi(x) d x\right| \leq & \frac{\zeta\left(u_{2}, u_{1}\right)}{4}\left(\int_{0}^{1} \frac{|1-2 \delta|}{h(\delta)} d \delta\right)^{\frac{1}{q}} \\
& \times\left(\left|\psi^{\prime}\left(u_{1}\right)\right|^{q}+\left|\psi^{\prime}\left(u_{2}\right)\right|^{q}\right) .
\end{aligned}
$$


Proof. We use Lemma 1 to show that

$$
\begin{aligned}
\left|\frac{\psi\left(u_{1}\right)+\psi\left(u_{1}+\zeta\left(u_{2}, u_{1}\right)\right)}{2}-\frac{1}{\zeta\left(u_{2}, u_{1}\right)} \int_{u_{1}}^{u_{1}+\zeta\left(u_{2}, u_{1}\right)} \psi(x) d x\right| & =\left|\frac{\zeta\left(u_{2}, u_{1}\right)}{2} \int_{0}^{1}(1-2 \delta) \psi^{\prime}\left(u_{1}+\delta \zeta\left(u_{2}, u_{1}\right)\right) d \delta\right| \\
& \leq \frac{\zeta\left(u_{2}, u_{1}\right)}{2} \int_{0}^{1}|1-2 \delta|\left|\psi^{\prime}\left(u_{1}+\delta \zeta\left(u_{2}, u_{1}\right)\right) d \delta\right| .
\end{aligned}
$$

Applying power-mean inequality, we get

$$
\begin{aligned}
\mid \frac{\psi\left(u_{1}\right)+\psi\left(u_{1}+\zeta\left(u_{2}, u_{1}\right)\right)}{2}-\frac{1}{\zeta\left(u_{2}, u_{1}\right)} \int_{u_{1}}^{u_{1}+\zeta\left(u_{2}, u_{1}\right)} & \psi(x) d x \mid \leq \frac{\zeta\left(u_{2}, u_{1}\right)}{2}\left(\int_{0}^{1}|1-2 \delta| d \delta\right)^{1-\frac{1}{q}} \\
& \times\left(\int_{0}^{1}|1-2 \delta|\left|\psi^{\prime}\left(u_{1}+\delta \zeta\left(u_{2}, u_{1}\right)\right)\right|^{q} d \delta\right)^{\frac{1}{q}} .
\end{aligned}
$$

Since $\left|\psi^{\prime}\right|^{q}$ is a $h$-Godunova-Levin preinvex, we obtain

$$
\begin{aligned}
\int_{0}^{1}|1-2 \delta|\left|\psi^{\prime}\left(u_{1}+\delta \zeta\left(u_{2}, u_{1}\right)\right)\right|^{q} d \delta & \leq \int_{0}^{1}\left(\frac{|1-2 \delta|}{h(\delta)}\left|\psi^{\prime}\left(u_{1}\right)\right|^{q}+\frac{|1-2 \delta|}{h(1-\delta)}\left|\psi^{\prime}\left(u_{2}\right)\right|^{q}\right) d \delta \\
& \leq \int_{0}^{1} \frac{|1-2 \delta|}{h(\delta)} d \delta\left(\left|\psi^{\prime}\left(u_{1}\right)\right|^{q}+\left|\psi^{\prime}\left(u_{2}\right)\right|^{q}\right) .
\end{aligned}
$$

Applying the basic calculus, we have $\int_{0}^{1}|1-2 \delta| d \delta=\frac{1}{2}$.

Corollary 4. Taking $\zeta\left(u_{2}, u_{1}\right)=u_{2}-u_{1}, h(\delta)=1$ and $q=1$ in inequality (9), we have

$$
\left|\frac{\psi\left(u_{1}\right)+\psi\left(u_{2}\right)}{2}-\frac{1}{u_{2}-u_{1}} \int_{u_{1}}^{u_{1}} \psi(x) d x\right| \leq \frac{u_{2}-u_{1}}{8}\left(\left|\psi^{\prime}\left(u_{1}\right)\right|+\left|\psi^{\prime}\left(u_{2}\right)\right|\right),
$$

which is similar to Theorem 2.2 repoted by Dragomir and Agarwal [4].

\section{Applications}

\subsection{Applications to Numerical Integration}

As mentioned in the introduction, the convexity can be applied to many areas of studies. Here, we give an example of how the $h$-Godunova-Levin convex and preinvex functions can be used to estimate the errors accumulated when using the trapezoidal formula for numerical integration.

Let $d$ be a division of the interval $\left[u_{1}, u_{2}\right]$, i.e., $d: u_{1}=v_{0}<v_{1}<\cdots<v_{n-1}<v_{n}=u_{2}$, of a given quadrature formula

$$
\int_{u_{1}}^{u_{2}} \psi(x) d x \cong T(\psi, d)+E(\psi, d),
$$

where

$$
T(\psi, d)=\sum_{i=0}^{n-1} \frac{\psi\left(v_{i}\right)+\psi\left(v_{i+1}\right)}{2}\left(v_{i+1}-v_{i}\right)
$$

is the trapezoidal formula. The associated approximation error is denoted by $E(\psi, d)$. 
Proposition 5. Let $\psi$ be a differentiable mapping on $S^{\circ}, u_{1}, u_{2} \in S^{\circ}$ with $u_{1}<u_{2}$. If $\left|\psi^{\prime}\right|$ is a $h$-Godunova-Levin preinvex on $\left[u_{1}, u_{1}+\zeta\left(u_{2}, u_{1}\right)\right]$, then for every division $d$ of $\left[u_{1}, u_{2}\right]$, we have

$$
\begin{aligned}
\left|E_{n}(\psi, d)\right| & \leq \frac{1}{2} \sum_{i=0}^{n-1}\left(v_{i+1}-v_{i}\right)^{2}\left(\left|\psi^{\prime}\left(v_{i}\right)\right|+\left|\psi^{\prime}\left(v_{i+1}\right)\right|\right) \int_{0}^{1} \frac{|1-2 \delta|}{h(\delta)} d \delta \\
& \leq \sum_{i=0}^{n-1}\left(v_{i+1}-v_{i}\right)^{2} \max \left\{\left|\psi^{\prime}\left(u_{1}\right)\right|,\left|\psi^{\prime}\left(u_{2}\right)\right|\right\} \int_{0}^{1} \frac{|1-2 \delta|}{h(\delta)} d \delta .
\end{aligned}
$$

Proof. We now apply Corollary (2) on the subinterval $\left[v_{i}, v_{i+1}\right](i=0,1,2, \ldots, n-1)$ of the division $d$. This gives the following:

$$
\left|\frac{\psi\left(v_{i}\right)+\psi\left(v_{i+1}\right)}{2}\left(v_{i+1}-v_{i}\right)-\int_{v_{i}}^{v_{i+1}} \psi(x) \mathrm{d} x\right| \leq \frac{\left(v_{i+1}-v_{i}\right)^{2}\left(\left|\psi^{\prime}\left(v_{i}\right)\right|+\left|\psi^{\prime}\left(v_{i+1}\right)\right|\right)}{2} \int_{0}^{1} \frac{|1-2 \delta|}{h(\delta)} d \delta .
$$

Since $\left|\psi^{\prime}\right|$ is $h$-Godunova-Levin preinvex, using the triangle inequality and summing the result over $i$ from 0 to $n-1$, we get

$$
\begin{aligned}
\left|T(\psi, d)-\int_{u_{1}}^{u_{2}} \psi(x) \mathrm{d} x\right| & \leq \frac{1}{2} \sum_{i=0}^{n-1}\left(v_{i+1}-v_{i}\right)^{2}\left(\left|\psi^{\prime}\left(x_{i}\right)\right|+\left|\psi^{\prime}\left(v_{i+1}\right)\right|\right) \\
& \leq \max \left\{\left|\psi^{\prime}\left(v_{i}\right)\right|,\left|\psi^{\prime}\left(v_{i+1}\right)\right|\right\} \sum_{i=0}^{n-1}\left(v_{i+1}-v_{i}\right)^{2} \int_{0}^{1} \frac{|1-2 \delta|}{h(\delta)} d \delta \\
& \leq \max \left\{\left|\psi^{\prime}\left(u_{1}\right)\right|,\left|\psi^{\prime}\left(u_{2}\right)\right|\right\} \sum_{i=0}^{n-1}\left(v_{i+1}-v_{i}\right)^{2} \int_{0}^{1} \frac{|1-2 \delta|}{h(\delta)} d \delta .
\end{aligned}
$$

The above inequality is an error bound of numerical integration obtained by $h$-Godunova-Levin preinvex. Choosing different functions of $h(\delta)$ in inequality (10) can give different results (see [6]).

\subsection{Applications to Special Means}

We finally use Hermite-Hadamard inequalities for $h$-Godunova-Levin preinvex function to form the inequalities for special means. Thus, the means of two positive numbers $u_{1}, u_{2}$, and $u_{1} \neq u_{2}$ can be considered as follows:

1. The arithmetic mean:

$A=A\left(u_{1}, u_{2}\right)=\frac{u_{1}+u_{2}}{2} ; u_{1}, u_{2} \in \mathbb{R}$, with $u_{1}, u_{2}>0$.

2. The generalized log-mean:

$$
L_{m}\left(u_{1}, u_{2}\right)=\left[\frac{u_{2}^{m+1}-u_{1}^{m+1}}{(m+1)\left(u_{2}-u_{1}\right)}\right]^{\frac{1}{m}}, m \neq-1,0
$$

The following propositions are obtained from the results in Section 4 and the above applications of special means.

Proposition 6. Let $0<u_{1}<u_{2}$, where $m \geq 2$, then we have

$$
\left|A\left(u_{1}^{m}, u_{2}^{m}\right)-L_{m}^{m}\left(u_{1}, u_{2}\right)\right| \leq \frac{m\left(u_{2}-u_{1}\right)}{2} A\left(\left|u_{1}^{m-1}\right|,\left|u_{2}^{m-1}\right|\right) \int_{0}^{1} \frac{|1-2 \delta|}{h(\delta)} d \delta .
$$

Proof. This inequality is obtained from Corollary (2) and applied on the $h$-Godunova-Levin preinvex function $\psi: \mathbb{R} \rightarrow \mathbb{R}, \psi(x)=x^{m}, m \geq 2$. 
Proposition 7. Let $0<u_{1}<u_{2}$, where $p>1, q=\frac{p}{p-1}$ and $m \geq 2$, then we get

$$
\left|A\left(u_{1}^{m}, u_{2}^{m}\right)-L_{m}^{m}\left(u_{1}, u_{2}\right)\right| \leq \frac{m\left(u_{2}-u_{1}\right)}{(p+1)^{\frac{1}{p}}} A\left(\left|u_{1}\right|^{\frac{(m-1) p}{p-1}},\left|u_{2}\right|^{\frac{(m-1) p}{p-1}}\right)^{\frac{1}{q}} \int_{0}^{1} \frac{1}{h(\delta)} d \delta .
$$

Proof. We derived this inequality from Corollary 3 applied to the $h$-Godunova-Levin preinvex function $\psi: \mathbb{R} \rightarrow \mathbb{R}$.

\section{Conclusions}

Since the Hermite-Hadamard type inequalities, due to their importance, can be found in many fields of study, the present study established new generalizations of such inequalities. Thus, two classes of function, $h$-Godunova-Levin and $h$-Godunova-Levin preinvex functions, along with some of their properties were established here. The applications to special means and numerical integration were also discussed in this study.

Author Contributions: O.A., writing—original draft preparation, visualization; A.K., writing-review and editing, supervision.

Funding: This research received no external funding

Acknowledgments: The authors would like to thank the referees and editors for their useful comments and remarks that improved the present manuscript substantially.

Conflicts of Interest: The authors declare no conflict of interest.

\section{References}

1. Dragomir, S.S. Two mappings in connection to Hadamard's inequalities. J. Math. Anal. Appl. 1992, 167, 49-56. [CrossRef]

2. Almutairi, A.; Kıliçman, A. New refinements of the Hadamard inequality on coordinated convex function. J. Inequal. Appl. 2019, 2019, 192. [CrossRef]

3. Dragomir, S.S. Lebesgue Integral Inequalities of Jensen Type for $\lambda$-Convex Functions. Armen. J. Math. 2018, 10, 1-19.

4. Dragomir, S.S.; Agarwal, R.P. Two inequalities for differentiable mappings and applications to special means of real numbers and to trapezoidal formula. Appl. Math. Lett. 1998, 11, 91-95. [CrossRef]

5. Robinson, S.M. An application of error bounds for convex programming in a linear space. SIAM J. Control 1975, 13, 271-273. [CrossRef]

6. Özdemir, M.E. Some inequalities for the s-Godunova-Levin type functions. Math. Sci. 2015, 9, 27-32. [CrossRef]

7. Pearce, C.E.; Pečarić, J. Inequalities for differentiable mappings with application to special means and quadrature formulae. Appl. Math. Lett. 2000, 13, 51-55. [CrossRef]

8. Kılıçman, A.; Saleh, W. Generalized preinvex functions and their applications. Symmetry 2018, 10, 493. [CrossRef]

9. Almutairi, O.; Kılıçman, A. New fractional inequalities of midpoint type via s-convexity and their application. J. Inequal. Appl. 2019, 2019, 1-19. [CrossRef]

10. Almutairi, O.; Kılıçman A. Generalized Integral Inequalities for Hermite-Hadamard-Type Inequalities via s-Convexity on Fractal Sets. Mathematics 2019, 7, 1065. [CrossRef]

11. Sarikaya, M.Z.; Saglam, A.; Yildirim, H. On some Hadamard-type inequalities for h-convex functions. J. Math. Inequal 2008, 2, 335-341. [CrossRef]

12. Dragomir, S.S.; Pecaric, J.; Persson, L.E. Some inequalities of Hadamard type. Soochow J. Math. 1995, 21, 335-341.

13. Godunova, E.K. Inequalities for functions of a broad class that contains convex, monotone and some other forms of functions. Numer. Math. Math. Phys. 1985, 138, 166.

14. Dragomir, S.S.; Pearce, C.E.M. On Jensen's inequality for a class of functions of Godunova and Levin. Periodica Math. Hung. 1996, 33, 93-100. [CrossRef] 
15. Dragomir, S.S.; Mond, B. On Hadamard's inequality for a class of functions of Godunova and Levin. Indian J. Math. 1997, 39, 1-10.

16. Radulescu, M.; Radulescu, S.; Alexandrescu, P. On the Godunova-Levin-Schur class of functions. Math. Inequal. Appl. 2009, 12, 853-862. [CrossRef]

17. Dragomir, S.S. Integral inequalities of Jensen type for $\lambda$-convex functions. Matematicki Vesnik 2016, 68, 45-57.

18. Varošanec, S. On h-convexity. J. Math. Anal. Appl. 2007, 326, 303-311. [CrossRef]

19. Ben-Israel, A.; Mond, B. What is invexity? ANZIAM J. 1986, 28, 1-9. [CrossRef]

20. Weir, T.; Mond, B. Preinvex functions in multiple objective optimization. J. Math. Anal. Appl. 1988, 136, $29-38$. [CrossRef]

21. Wu, S.H.; Baloch, I.A.; Işcan, I. On Harmonically-Preinvex Functions. J. Funct. Spaces 2017. [CrossRef]

22. Pavic, Z.; Wu, S.; Novoselac, V. Important inequalities for preinvex functions. J. Nonlinear Sci. Appl. 2016, 9, 3570-3579. [CrossRef]

23. Dragomir, S.S.; Fitzpatrick, S. The Hadamard inequalities for s-convex functions in the second sense. Demonstr. Math. 1999, 32, 687-696. [CrossRef]

24. Barani, A.; Ghazanfari, A.G.; Dragomir, S.S. Hermite-Hadamard inequality for functions whose derivatives absolute values are preinvex. J. Inequal. Appl. 2012, 2012, 247. [CrossRef]

(C) 2019 by the authors. Licensee MDPI, Basel, Switzerland. This article is an open access article distributed under the terms and conditions of the Creative Commons Attribution (CC BY) license (http:/ / creativecommons.org/licenses/by/4.0/). 